\title{
Pulmonary chondroma: A clinicopathological study of 29 cases and a review of the literature
}

\author{
DONG TIAN*, HONGYING WEN*, YU ZHOU* and MAOYONG FU \\ Department of Cardiothoracic Surgery, The Affiliated Hospital of North Sichuan Medical College, \\ Nanchong, Sichuan 637000, P.R. China
}

Received January 21, 2016; Accepted June 21, 2016

DOI: $10.3892 / \mathrm{mco} .2016 .945$

\begin{abstract}
The present retrospective study was designed to review the clinicopathological features and outcome of surgical treatment of pulmonary chondroma, and to accumulate data for the clinical diagnosis and therapy. The clinicopathological data from 29 patients, aged between 38- and 78-years-old, with pulmonary chondroma who underwent surgical operation between July 2003 and June 2015 were reviewed. Of these patients, 18 exhibited no clinical symptoms, 7 were characterized by coughing, hemoptysis, shortness of breath and other symptoms and only 3 patients exhibited chest pain as the predominant symptom. The average size of the neoplasms was $3.6 \mathrm{~cm}$. All patients were pathologically diagnosed. Operative time was $126 \pm 22 \mathrm{~min}$, the mean intraoperative blood loss was $82 \pm 23 \mathrm{ml}$ and the drainage duration was $3.1 \pm 1.8$ days. A total of 6 postoperative complications were noted. The patients were followed-up for 2-135 months. A total of 23 patients were alive without recurrence, 4 patients succumbed to mortality, 2 patients were lost at follow-up. Pulmonary chondroma is a rare benign tumor of the lung. The clinical symptoms were concealed and often misdiagnosed as a tuberculosis tumor, hamartoma, peripheral lung cancer or a single metastatic tumor. Complete resection was the best treatment providing patients with a good prognosis. After definite diagnosis, it is necessary to exclude Carney's triad.
\end{abstract}

\section{Introduction}

Chondroma is a benign tumor, originating in chondrocytes. It is common in long bone, but rarely occurs in the lung parenchyma. The clinicopathological features of pulmonary

Correspondence to: Dr Maoyong Fu, Department of Cardiothoracic Surgery, The Affiliated Hospital of North Sichuan Medical College, Nanchong, Sichuan 637000, P.R. China

E-mail: fumaoyongmd@126.com

*Contributed equally

Key words: pulmonary chondroma, clinicopathological features, surgical treatment chondroma has been rarely reported $(1,2)$. Bateson (3) distinguished endobronchial chondroma from pulmonary chondroma based on the histogenesis of lung chondroma. The former grows into the bronchial lumen and accounts for $<25 \%$ of all benign cartilage neoplasms of the lung. The latter grows into lung parenchyma and has an incidence of about $0.1 \%$ of all benign lung tumor types. Between July 2003 and June 2015, 29 patients with pulmonary chondroma underwent surgical operation at The Affiliated Hospital of North Sichuan Medical College (Nanchong, China). All patients were pathologically diagnosed. The present paper aimed to summarize the clinicopathological features and the outcome of surgical treatment of pulmonary chondroma. Additionally, the present study clarified the differences between pulmonary chondroma and other benign pulmonary tumor.

\section{Materials and methods}

Clinical data. The present study was approved by the Ethics Committee of North Sichuan Medical College (Nanchong, China). All patients provided written informed consent. The clinical data for each of the 29 patients is shown in Table I. A total of 29 patients were pathologically diagnosed pulmonary chondroma, including 16 males and 13 females, with an average age of 57 years (range, 39-78-years-old). Of these patients, 18 exhibited no clinical symptoms and the pulmonary chondroma was detected by routine medical examination, 7 patients were characterized by coughing, hemoptysis, shortness of breath and other symptoms, only 3 patients presented with chest pain as the predominant symptom and 1 patient with esophageal cancer was identified by preoperative examination. Physical examination revealed only 3 patients with low breath sounds and others without obvious abnormalities. Lung tumor-like lesions, nodules and varying degrees of calcification was demonstrated by chest computed tomography (CT) scan (Fig. 1). No significant enlargement of the lymph nodes and pulmonary cavity was observed. Pulmonary masses of 9 patients were in the right lower lobe, 2 were in the right upper lobe, 3 were in the right middle lobe, 11 were in the left upper lobe and 4 were in the left lower lobe. The mean tumor diameter was $3.6 \mathrm{~cm}$, ranging between $1.0 \mathrm{~cm}$ and $8.5 \mathrm{~cm}$. The edge of the 8 cases were rough and the rest were smooth. No obvious abnormalities were revealed by other routine preoperative examinations, 
including head CT scan, radionuclide bone scan and abdominal ultrasound. Preoperatively, 13 patients were considered hamartoma, 14 patients were considered benign nodules, only 2 patients with chest wall adhesion were suspected of malignant infiltration and no patient was diagnosed pulmonary chondroma.

Operative technique. The thoracotomy pneumonectomy was performed under general anesthesia with single-lung ventilation, which may be accomplished with double-lumen endotracheal tubes. The patients were placed in the lateral decubitus position with the upper arm suspended on a crossbar. Firstly, a $1.5 \mathrm{~cm}$ incision was placed in the seventh intercostal space at mid axillary line. The pleural cavity was entered to explore the lesion and surrounding structures. Another incision ( 7-9 cm long) was made in the fourth intercostal space under the armpit if there was no adhesion, or else anterolateral incision was used. During conventional thoracotomy, the mass revealed no malignant cells by intraoperative frozen section examination and was therefore confirmed as benign tumors.

Statistical analysis. The data were analyzed using SPSS 22.0 (IBM SPSS, Chicago, IL, USA) and the results were presented as the mean \pm standard deviation.

\section{Results}

A total of 29 patients were pathologically diagnosed with pulmonary chondroma, including 16 males and 13 females, with an average age of 57 years (range, 39-78 years). Of these patients, 18 exhibited no clinical symptoms and the pulmonary chondroma was detected by routine medical examination, 7 were characterized by coughing, hemoptysis, shortness of breath and other symptoms, 3 patients presented with chest pain as the predominant symptom and 1 patient with esophageal cancer was identified by preoperative examination. Physical examination revealed only 3 patients with low breath sounds and others without obvious abnormalities. Lung tumor-like lesions, nodules and varying degrees of calcification was demonstrated by chest computed tomography (CT) scan (Fig. 1). No significant enlargement of the lymph nodes and pulmonary cavity was observed. Pulmonary masses of 9 patients were found in the right lower lobe, 2 were in the right upper lobe, 3 were in the right middle lobe, 11 were in the left upper lobe and 4 were in the left lower lobe. The mean tumor diameter was $3.6 \mathrm{~cm}$, ranging between 1 and $8.5 \mathrm{~cm}$. The edge of the 8 cases were rough and the rest were smooth. No obvious abnormalities were revealed by other routine preoperative examinations, including head CT scan, radionuclide bone scan and abdominal ultrasound. Preoperatively, 13 patients were considered hamartoma, 14 patients were considered benign nodules, 2 patients with chest wall adhesion were suspected of malignant infiltration and no patient was diagnosed with pulmonary chondroma.

A total of 11 patients underwent lobectomy (Fig. 2), 17 patients underwent segmentectomy and 1 patient used lump stripping. They were postoperatively pathologically diagnosed as pulmonary chondroma (Fig. 3). All the lymph nodes were reactive hyperplasia. Carney's triad was excluded by abdominal magnetic resonance imaging (MRI)

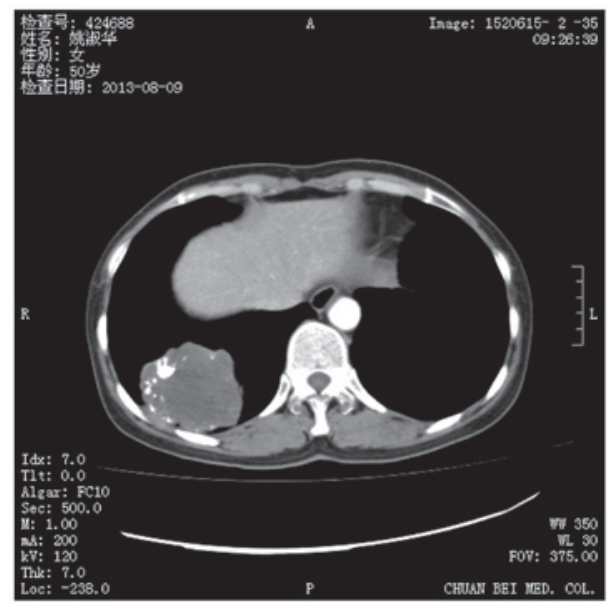

Figure 1. Contrast-enhanced computed tomography revealed a large inhomogeneous density mass with varying degrees of calcification.
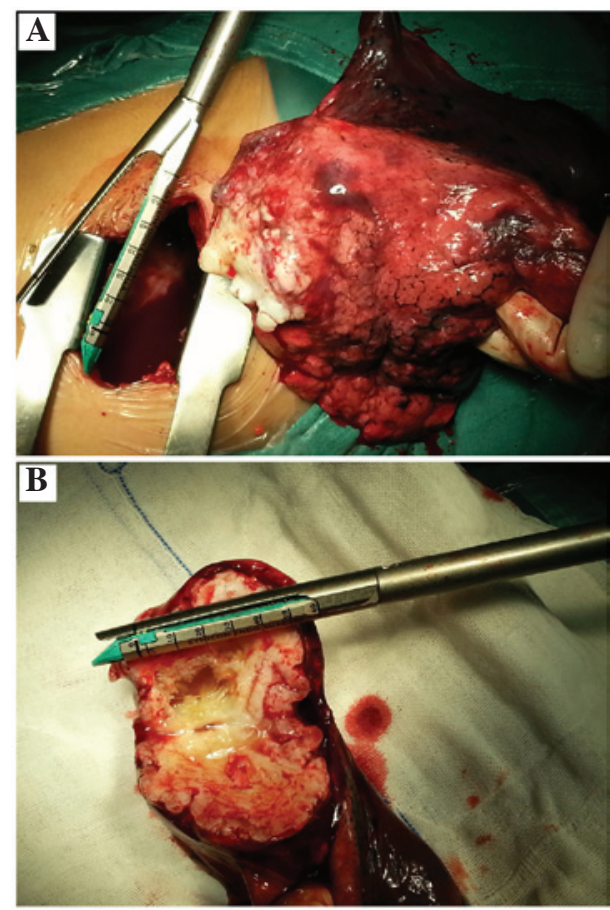

Figure 2. Images of the resected mass. (A) Patients with pulmonary chondroma underwent lobectomy intraoperatively. (B) The mass was pale and translucent, hard, and lobulated on the lateral section.

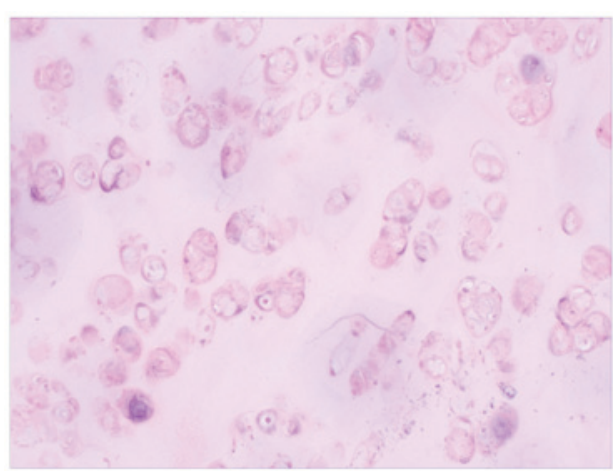

Figure 3. Hematoxylin and eosin staining of tumor cells. The tumor was revealed to be formed by differentiation of mature cartilage tissue wrapped around by cartilage matrix (magnification, x200). 
Table I. Clinical data.

\begin{tabular}{|c|c|c|c|c|c|c|c|c|c|}
\hline $\begin{array}{l}\text { Patient } \\
\text { no. }\end{array}$ & $\begin{array}{c}\text { Age } \\
\text { (years) }\end{array}$ & Gender & $\begin{array}{l}\text { Size } \\
(\mathrm{cm})\end{array}$ & $\begin{array}{l}\text { Operative } \\
\text { time (min) }\end{array}$ & $\begin{array}{c}\text { Blood } \\
\text { loss (ml) }\end{array}$ & $\begin{array}{l}\text { Drainage } \\
\text { (days) }\end{array}$ & Follow-up & Position & $\begin{array}{c}\text { Preoperative } \\
\text { diagnosis }\end{array}$ \\
\hline 1 & 51 & M & 2.3 & 64 & 45 & 3 & Alive without recurrence & Right lower lobe & Hamartoma \\
\hline 2 & 43 & $\mathrm{~F}$ & 4.6 & 78 & 30 & 4 & Alive without recurrence & Right lower lobe & Benign nodules \\
\hline 3 & 38 & M & 5.2 & 155 & 10 & 2 & $\begin{array}{l}\text { Succumbed to } \\
\text { non-neoplastic diseases }\end{array}$ & Right upper lobe & Hamartoma \\
\hline 4 & 62 & M & 1.4 & 92 & 100 & 2 & Alive without recurrence & Right middle lobe & Benign nodules \\
\hline 5 & 53 & M & 1 & 146 & 60 & 3 & Alive without recurrence & Right lower lobe & Hamartoma \\
\hline 6 & 68 & $\mathrm{~F}$ & 3.9 & 104 & 5 & 3 & Alive without recurrence & Left lower lobe & Hamartoma \\
\hline 7 & 56 & $\mathrm{~F}$ & 4.4 & 132 & 50 & 3 & Alive without recurrence & Right upper lobe & Benign nodules \\
\hline 8 & 57 & $\mathrm{~F}$ & 2.3 & 141 & 120 & 2 & $\begin{array}{l}\text { Succumbed to } \\
\text { non-neoplastic diseases }\end{array}$ & Right lower lobe & Hamartoma \\
\hline 9 & 72 & $\mathrm{~F}$ & 6.8 & 137 & 80 & 2 & Alive without recurrence & Left upper lobe & Benign nodules \\
\hline 10 & 52 & M & 4.1 & 48 & 30 & 2 & Alive without recurrence & Right lower lobe & Hamartoma \\
\hline 11 & 47 & $\mathrm{~F}$ & 2.6 & 164 & 90 & 4 & Lost & Right lower lobe & Malignant tumor \\
\hline 12 & 78 & M & 6.9 & 143 & 180 & 3 & Alive without recurrence & Left upper lobe & Benign nodules \\
\hline 13 & 64 & M & 1.8 & 156 & 40 & 4 & Alive without recurrence & Left upper lobe & Benign nodules \\
\hline 14 & 73 & $\mathrm{~F}$ & 3 & 123 & 30 & 2 & Lost & Right middle lobe & Hamartoma \\
\hline 15 & 66 & M & 2.5 & 157 & 75 & 5 & Alive without recurrence & Left lower lobe & Benign nodules \\
\hline 16 & 69 & $\mathrm{~F}$ & 5 & 129 & 350 & 2 & Alive without recurrence & Right lower lobe & Benign nodules \\
\hline 17 & 64 & M & 8.5 & 92 & 40 & 3 & $\begin{array}{l}\text { Succumbed to } \\
\text { non-neoplastic diseases }\end{array}$ & Right middle lobe & Benign nodules \\
\hline 18 & 57 & $\mathrm{~F}$ & 3.8 & 201 & 60 & 2 & Alive without recurrence & Left upper lobe & Benign nodules \\
\hline 19 & 52 & $\mathrm{~F}$ & 3.2 & 107 & 80 & 8 & Alive without recurrence & Left lower lobe & Hamartoma \\
\hline 20 & 55 & M & 1.9 & 215 & 240 & 3 & Alive without recurrence & Right lower lobe & Hamartoma \\
\hline 21 & 58 & M & 1.8 & 104 & 30 & 4 & Alive without recurrence & Left upper lobe & Malignant tumor \\
\hline 22 & 46 & $\mathrm{~F}$ & 3.7 & 106 & 50 & 3 & $\begin{array}{l}\text { Succumbed to } \\
\text { non-neoplastic diseases }\end{array}$ & Left upper lobe & Hamartoma \\
\hline 23 & 70 & $\mathrm{~F}$ & 2.2 & 192 & 40 & 3 & Alive without recurrence & Left lower lobe & Benign nodules \\
\hline 24 & 51 & M & 3.2 & 104 & 45 & 3 & Alive without recurrence & Left upper lobe & Benign nodules \\
\hline 25 & 48 & M & 1.2 & 174 & 100 & 2 & Alive without recurrence & Left upper lobe & Hamartoma \\
\hline 26 & 59 & $\mathrm{~F}$ & 7.4 & 117 & 80 & 5 & Alive without recurrence & Right lower lobe & Hamartoma \\
\hline 27 & 54 & M & 2.3 & 94 & 100 & 3 & Alive without recurrence & Left upper lobe & Benign nodules \\
\hline 28 & 43 & M & 4.2 & 89 & 160 & 2 & Alive without recurrence & Left upper lobe & Benign nodules \\
\hline 29 & 47 & M & 3.2 & 90 & 55 & 3 & Alive without recurrence & Left upper lobe & Hamartoma \\
\hline
\end{tabular}

M, male; F, female.

and gastroscopy. No mortality occurred during surgery. The operative duration ranged between 48 and $215 \mathrm{~min}$ (mean, 126 $\pm 22 \mathrm{~min}$ ). The estimated blood loss ranged between 5 and $350 \mathrm{ml}$ (mean, $82 \pm 23 \mathrm{ml}$ ). Additionally, no patient required a blood transfusion. All patients, with the exception of 5 patients, had an uneventful postoperative course $(82.8 \%)$. Of the five complications, two were postoperative encapsulated pleural effusion and three were pulmonary infection. These 5 patients recovered well following percutaneous catheter drainage by CT-guided and anti-infection therapy. The drainage duration ranged between 2 and 8 days (mean, 3.1 \pm 1.8 days) and the postoperative hospital duration ranged between 4 and 13 days (mean, 4.0 2.1 days). Patients were followed-up between 2 and 135 months. During follow-up, 23 patients were alive without recurrence, 1 patient succumbed to esophageal cancer after 19 months post-surgery, 3 patients succumbed to other diseases. A total of 2 patients were lost during follow-up.

\section{Discussion}

Pulmonary chondroma is often originated from ectopic cartilage of lung tissue during embryonic development. Chondrocytes in other parts of the tissues flowed into the lungs by bloodstream. Connective tissues, reticulocytes developed into original direction by certain stimulate conditions, became the embryo of mesenchymal tissue, and then developed into chondrocytes. These are theoretical speculations (2).

The tumor tissues were pale and translucent, hard and lobulated on the lateral section. Under the microscope, the tumors were observed to be formed by differentiation of mature cartilage tissue, wrapped around the cartilage matrix. Cartilage tissues can be hyaline cartilage, elastic cartilage and fibrous cartilage, or diverse cartilage mixed together without other mesenchymal tissue components, abnormal mitotic and adipose tissues. Chondrocytes can encounter calcification, ossification and mucoid degeneration (4). 
A few case reports have identified pulmonary chondroma $(1,2)$. Only $0.04 \%$ of lung neoplasms were identified to be pulmonary chondroma (3). Pulmonary chondroma was common in adult females of 40-50-years-old and neonatal cases were occasionally reported (2). However, in the present group, 16 males and 13 females with an average age of 57 years (range, 39-78-years-old), different from the literature $(2,4,5)$. Pulmonary chondroma may occur in each pulmonary lobe, particularly the right lower lobe. Of the 29 patients, 9 exhibited pulmonary chondroma located in the right lower lobe and 15 were located in left lung, also inconsistent with previous literature. The mean diameter of pulmonary chondroma was $2.8 \mathrm{~cm}$ and was usually asymptomatic (4). In the present study, the average diameter was $3.6 \mathrm{~cm}(1.0-8.5 \mathrm{~cm})$. Of the patients, 18 presented without any clinical symptoms, 1 with esophageal cancer was identified by preoperative examination. The symptoms of pulmonary chondroma depended on the size and location of the tumor. If the bronchus were oppressed, atelectasis was caused. Respiratory symptoms, including cough with sputum or hemoptysis and shortness of breath, can occur $(6,7)$. In the present study, 7 cases $(13.8 \%)$ occurred. In addition, 3 patients exhibited chest pain. From the CT scan, the tumor size in all three patients were larger and exhibited chest wall invasion. Intraoperatively, the tumor shrunk without invasion into the chest wall. As a result of the compression of intercostal nerves, the patients encountered chest pain (8).

Generally, pulmonary chondroma is one of the clinical features of Carney's triad. Carney et al (9) first reported the disease in 1977. This study included gastrointestinal stromal tumor, pulmonary chondroma, extra-adrenal paraganglioma. If $2 / 3$ clinical features are present, it can be diagnosed as Carney's triad (9). Clinically, 2 features were often observed in $~ 78 \%$ of patients (5). Generally, gastrointestinal stromal tumors merged with pulmonary chondroma occurred more often in $\sim 53 \%$. The syndrome is rare and predominantly affects young women; however, the etiology remains unknown. Surgical resection was the predominant treatment, which has a higher postoperative survival. A total of 104 patients with Carney's triad were reported to have survival rates of 10 and 40 years, for 100 and $73 \%$, respectively (10). For young women, a gastrointestinal stromal tumor or pulmonary chondroma should be considered as one of the clinical features of Carney's triad. In addition, gastrointestinal stromal tumors and extra-adrenal paraganglioma in Carney's triad were potentially fatal, and eliminating the syndrome for patients with pulmonary chondroma was necessary. The correct diagnosis for patients of Carney's triad is essential to provide the appropriate treatment and result in a good prognosis. To exclude Carney's triad, the 29 patients in the present study underwent abdominal MRI examination and gastroscopy 3 months after surgery and no abnormalities were detected. As a result of the heterochrony of the three tumors, lifelong follow-up of patients with pulmonary chondroma is necessary.

A chest X-ray, enhanced CT scan of the chest or MRI made it easier to identify the benign or malignant calcification lumps. The CT is more commonly used, often displaying as round or oval nodules, moderate soft tissue density, inhomogeneous density with calcification and clear boundaries. Tumor diameter often ranged between 1.0 and $4.0 \mathrm{~cm}$ with mild lobulation. No glitches, satellite lesions or enlarged lymph nodes at the hilus of lung or mediastinum were observed (11). In the present study, patients with enhanced CT scan presented similarly with those in the literature. Ultimately, pathological diagnosis of the tumor is required.

Pulmonary chondroma can be easily misdiagnosed as tuberculosis tumor, hamartoma (particularly cartilage hamartoma) and peripheral lung cancer. In the present study, varying degrees of calcification were observed in all cases. A total of 13 patients were considered to have hamartoma, 14 patients were considered to exhibit benign nodules, 2 patients with chest wall adhesion were suspected of malignant infiltration and all patients were not diagnosed as pulmonary chondroma.

Patients with a previous history of tuberculosis was usually considered to have pulmonary tuberculoma (12). Tuberculoma was often located in the dorsal segment of the lower lobe. Calcifications and cavity were found in the lesion and were characterized by circular structures by enhanced CT scan. The most common benign tumor of the lung was hamartoma, which accounted for 5-10\% of solitary pulmonary nodules (13). Calcification looked like popcorn or the image of fat density with enhanced CT scan and allowed the differentiation of points of hamartoma. Notably, calcification of pulmonary chondroma was smaller, mostly point-like or scaly. In the present study, 13 patients $(44.8 \%)$ were misdiagnosed as having hamartoma. For older individuals who smoked perennially, a cough with sputum and blood may easily be misdiagnosed as peripheral lung cancer. Enhanced CT scans revealed a burr-like structure of the tumor, pleural indentation and inhomogeneous enhancement often accompanied by enlarged lymph nodes at the hilus of the lung or mediastinum (14). Although the average age in the present study was older, varying degrees of calcification were revealed in the CT. Only 2 patients with chest wall adhesion were suspected of malignant infiltration. In addition, previous medical history, including primary malignant tumor and solitary pulmonary metastases, was difficult to identify with non-calcified pulmonary chondroma.

Although primary lung tumors are benign tumors of cartilage, there remains the possibility of malignant transformation. Mei et al (15) reported a case of giant primary mesenchymal chondrosarcoma of the lung, followed-up for 6 months and this patient succumbed to tumor recurrence and metastasis (15). Certain patients succumb to Carney's triad as a result of malignant alteration of lesions. Therefore, the patients with Carney's triad must be given a medical check periodically. It was more difficult to identify malignant pulmonary chondroma with chondrosarcoma on the image presentations. Surgical resection was the preferred treatment for pulmonary chondroma. With the advantages of less trauma and faster recovery, thoracoscopy or subaxillary minithoracotomy was used as the preferred treatment (16). In the present study, 23 cases were completely resected without residual tumor or postoperative recurrence.

Pulmonary chondroma is a rare benign tumor of the lung and the etiology remains unknown. It grows slowly with hidden clinical symptoms, often identified by routine medical examination. Pulmonary chondroma can be easily misdiagnosed as a tuberculosis tumor, hamartoma (particularly cartilage hamartoma) and peripheral lung cancer. As a result of the possibility of the malignant transformation, complete surgical resection is the best treatment. Pulmonary chondroma 
are possibly an initial clinical presentation of Carney's triad; therefore, following the diagnosis of pulmonary chondroma, further examination is required to exclude the Carney's triad.

\section{Acknowledgements}

The authors would like to thank Dr Xiaoguang Guo of the Department of Pathology, Nanchong Central Hospital, The Second Clinical Institute of North Sichuan Medical College (Nanchong, China) for the support and assistance.

\section{References}

1. Ishii H, Akiba T, Marushima H, Kanetsuna $\mathrm{Y}$ and Morikawa $\mathrm{T}$ : A case of bilateral multiple pulmonary chondroma: Necessity of follow-up for Carney's triad. Gen Thorac Cardiovasc Surg 60 534-536, 2012

2. Hoekstra MO, Bertus PM, Nikkels PG and Kimpen JL: Multiple pulmonary chondromata. A rare cause of neonatal respiratory distress. Chest 105: 301-302, 1994.

3. Bateson EM: Histogenesis of intrapulmonary and endobronchial hamartomas and chondromas (cartilage-containing tumours): A hypothesis. J Pathol 101: 77-83, 1970.

4. Rodriguez FJ, Aubry MC, Tazelaar HD, Slezak J and Carney JA Pulmonary chondroma: A tumor associated with Carney triad and different from pulmonary hamartoma. Am J Surg Pathol 31: 1844-1853, 2007.

5. Carney JA: Gastric stromal sarcoma, pulmonary chondroma, and extra-adrenal paraganglioma (Carney Triad): Natural history, adrenocortical component, and possible familial occurrence. Mayo Clin Proc 74: 543-552, 1999.
6. Ammar A, El Hammami S and Sellami Kamoun N: Chondromas-a rare lung tumour: Rev Mal Respir 22: 826-827, 2005 (In French).

7. Silva VA, Kataguiri P, Trufelli DC, Matos LL, Neves-Pereira JC and Campos JR: Pulmonary hamartoma as a differential diagnosis of breast cancer metastasis: Case report. J Bras Pneumol 33: 738-742, 2007 (In Portuguese).

8. Allan JS: Rare solitary benign tumors of the lung. Semin Thorac Cardiovasc Surg 15: 315-322, 2003.

9. Carney JA, Sheps SG, Go VL and Gordon H: The triad of gastric leiomyosarcoma, functioning extra-adrenal paraganglioma and pulmonary chondroma. N Engl J Med 296: 1517-1518, 1977.

10. Zhang L, Smyrk TC, Young WF Jr, Stratakis CA and Carney JA: Gastric stromal tumors in Carney triad are different clinically, pathologically, and behaviorally from sporadic gastric gastrointestinal stromal tumors: Findings in 104 cases. Am J Surg Pathol 34: 53-64, 2010.

11. Strano S, Ouafi L, Baud M and Alifano M: Primary chordoma of the lung. Ann Thorac Surg 89: 302-303, 2010.

12. Fain O: Pulmonary tuberculoma. Rev Prat 55: 1751, 2005 (In French).

13. Dragoumis DM, Boudalaki ES, Assimaki AS and Tsiftsoglou AP: Pulmonary hamartoma masquerading lung metastasis in a woman with inflammatory breast cancer. Breast J 18: 486-488, 2012.

14. Yokota H, Shigeta A, Edo H and Niijima M: Peripheral lung cancer effectively diagnosed with virtual bronchoscopy. Nihon Naika Gakkai Zasshi 96: 781-783, 2007 (In Japanese).

15. Mei B, Lai YL, He GJ, Shou YN and Liu J: Giant primary mesenchymal chondrosarcoma of the lung: Case report and review of literature. Ann Thorac Cardiovasc Surg 19: 481-484, 2013.

16. Ludwig C, Zeitoun $\mathrm{M}$ and Stoelben E: Video-assisted thoracoscopic resection of pulmonary lesions. Eur J Surg Oncol 30: $1118-1122,2004$ 